\title{
Barriers to Communication About Complementary and Alternative Medicine With Patients: A Qualitative Study
}

\author{
Hsiao-Yun Chang ${ }^{1}$, Tzu-Fang $\mathrm{Su}^{2} \&$ Robert McCreary Mannino ${ }^{1}$ \\ ${ }^{1}$ School of Nursing, Fooyin University, Kaohsiung, Taiwan \\ ${ }^{2}$ Department of Metabolism, Shing-Hou Hospital, Kaohsiung, Taiwan \\ Correspondence: Hsiao-Yun Chang, 151 Jinxue Rd., Daliao Dist., Kaohsiung City 83102, Taiwan. Tel: \\ 886-98-650-2206. E-mail: chang369@gmail.com
}

Received: June 13, 2019 Accepted: August 7, 2019 Online Published: August 13, 2019

doi:10.5539/gjhs.v11n10p43

URL: https://doi.org/10.5539/gjhs.v11n10p43

\begin{abstract}
Background: The purpose of this study is to explore the reasons for nurses' reluctance to communicate with patients regarding the use of complementary and alternative medicines (CAM) and to determine ways of improving this communication.
\end{abstract}

Methods: A qualitative study using focus group interviews was conducted with 54 nurses participated in eight focus groups. Interview data were transcribed verbatim using framework analysis. Ethical approval was obtained from the Research Ethics Board.

Results: The themes were related to the reasons for nurses' reluctance to communicate with patients were: (1) "the scope of nursing practice regarding CAM is unclear", (2) "lack of CAM competencies in communication", and (3) "unsupportive workplace culture in the communication of CAM". The strategies for improving the communication were: (1) "awareness of the needs for CAM education", (2) "engagement of interdisciplinary teamwork for CAM practice", and (3) "establishment of an organizational standard for CAM practice".

Conclusions: This study provided new insights into the barriers to communication regarding CAM use with patients from the nurses' perspectives, and helped identify the ways of improving this communication to advance the practice of nursing.

Keywords: complementary and alternative medicine, communication, nurse, qualitative study

\section{Introduction}

Complementary and Alternative Medicine (CAM) refers to practices, approaches, knowledge, and beliefs incorporating biologically-based therapies, mind-body therapies, manipulative techniques, energy therapies and whole systems of care that are not generally considered as a part of conventional medicine (National Center for Complementary and Integrative Health (NCCIH), 2018). These therapies are rarely taught to healthcare students in school, and are not generally available at hospitals in Taiwan. The prevalence of CAM use among adult population in Taiwan is high and as a national study indicates that $86.9 \%$ people had used at least one modality in the past year (Yeh, Lin, Chen, Wang, \& Huang, 2015). Rates of using both CAM and conventional medicine among patient population are also widespread, such as $48 \%$ for patients with Parkinson's disease (Chen et al., 2018) and 54.1\% for patients with cancer (Wu, Tai, Tai, \& Chien, 2019). Interestingly, one study on patients with neurological problems found that only $17.4 \%$ admitted to using one or more types of CAM; however, the actual usage rate was 41.6\% after the follow-up questions (Davis, Oh, Butow, Mullan, \& Clarke, 2012). Foley et al. (2019) reviewed the disclosure rates of biologically-based CAM use to conventional healthcare professionals ranging from 7\% to $80 \%$ with average of $33 \%$ disclosure rate. The disclosure rate is even lower in countries with medical pluralism, which involves conventional medicine as a dominant system in coexistence with traditional medicine: $26 \%$ in Taiwan (H. Y. Chang, H. L. Chang, \& Siren, 2013) and $0.5 \%$ in Iraq (Hwang et al., 2016). The reasons for nondisclosure among patients have been reported as it's irrelevance to their conventional care, feeling that the natural products were safe, informally consulting acquaintances who are health professionals, belief healthcare professionals lacked CAM knowledge and time, apprehension regarding the development of negative relationships with healthcare professionals, and negative reactions from healthcare professionals, such as anticipation of a physician's disapproval, disinterest, inability to help, or lack of inquiry(Chang et al., 2013; Foley et al., 2019; Jou 
\& Johnson, 2016).

In fact, the most common reason reported by patients are fear of a negative reaction and lack of inquiry from their healthcare professionals (Foley et al., 2019). In Australia, Hall et al. (2018) interviewed 19 registered nurses on how to communicate with patients about CAM and found that many nurses elaborated "the barriers to CAM communication" (P. 285). The definition of CAM communication is an interactive process in which a patient-healthcare professional dialogue involves gathering information about patient's CAM use, addressing patient's concerns, providing a reliable response concerning this use and assisting patients in decisions about the safety of CAM use (Frenkel \& Cohen, 2014). A review study focusing on nurses' knowledge and attitudes regarding CAM and their ability to communicate about CAM indicated that $66.4 \%$ of nurses had positive attitudes towards CAM, 77.4\% did not possess a comprehensive understanding of CAM, and $47.3-67.7 \%$ of them reported feeling uncomfortable discussing CAM (H. Y. Chang \& H. L. Chang, 2015). The high non-disclosure rate of CAM use among patients and the hesitant attitude toward communication about CAM among nurses may put patients at greater risk of a life-threatening interaction between CAM and conventional medicine or other risks such as delay in seeking conventional diagnosis/care, not receiving an appropriate treatment or simply wasting money on ineffective CAM etc. (Firkins et al., 2018).

The risk associated with this non-communication is that concurrent use of CAM with pharmacotherapy may cause an adverse herb-drug interaction. A study found that $85.1 \%$ of those who used biological-based CAM (vitamins, trace elements, supplements, or phytotherapeutics) were at some risk of interactions with conventional treatments (interferon, radiotherapy, chemotherapy, BRAF-inhibitor, or other tyrosine kinase inhibitors and ipilimumab) (Loquai et al., 2017). Evidence has shown that patient-healthcare professional communication about the use of CAM is associated with higher patient satisfaction and lower potential drug-herb interaction (Davis et al., 2012). However, nurses' barriers to communication among nurses about CAM use with patients are seldom discussed in literature, and literature is particularly lacking on how to improve this communication gap in care.

\subsection{Purpose}

This study has tended to focus on the reasons for nurses' reluctance to communicate with patients regarding CAM use, their concerns in dealing with patients' CAM use, and finding ways of improving this communication.

\section{Methods and Materials}

\subsection{Study Design}

A qualitative study design using focus group interviews was employed to understand the perceptions, feelings, and thoughts of nurses on communicating with patients regarding CAM use. The focus group research technique is an effective approach used to collect qualitative data, particularly to enhance communication about a specific subject (Reed \& Payton, 1997). This study used this formal method to explore reasons for nurses' reluctance to communicate with patients regarding complementary and alternative medicine and to find ways to improve this communication.

\subsection{Sample and Setting}

Participants were recruited from different hospitals in the South of Taiwan. To ensure that groups consisted of persons capable of providing the highest-quality discussion about the topic being researched, certain eligibility criteria were used to screen the volunteers: 1) above 20 years of age and 2) had more than one year of work experience as a registered nurse. Participants varied in terms of field, professional experience, age, official position, and types of medical institutions. A total of eight focus groups was conducted with five to eight persons in each group to obtain an adequate range and depth of data.

\subsection{Instruments}

An interview guide was developed by the researchers and the content covered the following sections: 1) introduction: to briefly explain the purpose of this session and the surroundings; 2) a warm-up: to discuss general issues related to CAM; 3 ) a section on details: to identify important information about barriers to the discussion about CAM with patients and to explore acceptable solutions for bridging this communication gap; 4) a key content section: to facilitate further in-depth discussion to ensure the discussion thoroughly; 5) summary: to give participants an opportunity to share any information that they may have forgotten. This structured the interview around several focal questions and was designed to cover the main aspects of the research question. The interview guide was reviewed by a panel of experts: two experienced qualitative researchers, two professors in CAM education, and two nurses. 


\subsection{Data Collection}

At initial contact, the purposes of the study and the planed use of the information were explained to participants who met the inclusion criteria and arrangements were made after they gave consent. The group discussions lasted approximately 90-120 minutes, and were led by a moderator. Interview data were collected in the following three ways: 1) audio-recording, 2) notes taken during and after the interview, and 3) observational notes taken by the session observer. After each focus group, the observers and moderator remained in the room for 15 to 20 minutes for a post-group discussion. Data collection was terminated when thematic saturation was achieved, wherein new interviews produced no new significant themes.

\subsection{Data Analysis}

The initial interview and field notes were fully transcribed. The principle of framework analysis was used, including familiarization (identifying a thematic framework) and indexing (charting, mapping, and interpretation) (Rabiee, 2004). Two researchers analyzed the data separately and held discussions after each step. In the familiarization stage, researchers reviewed data by listening to the tapes and reading the transcripts and observational notes several times, and summary notes written during the post-group discussion. The next stage of identifying a thematic framework entailed writing memos in the margin of the text, such as short phrases, idea, or concepts, which arose from the texts and began forming categories. Next, the researchers conducted indexing by shifting and managing the data from the original context by comparing and contrasting the relevant information and re-organizing it into a newly-developed thematic matrix. The final stage of analysis was to map and interpret the coded data related to the research purpose. Data analysis ended when no new information emerged about a category and removing new data from the present data did not affect the analysis. After the analysis was complete, English translations from Chinese were created for publication. The translations were done by a professional translator.

\subsection{Methodological Rigor}

Lincoln and Guba's (1985) four criteria of trustworthiness were employed, namely, credibility, confirmability, dependability, and transferability. Confirmability and dependability were ensured in this study by using verbatim recording and auditing the transcripts and notes written during the study process to manage the data. Additionally, members of research committees provided feedback throughout the study. Credibility was ensured after completion of raw data analysis, as the researchers summarized the key points and asked 10 participants to confirm the findings. For dependability, the qualitative findings were compared with literature to identify consistencies and discrepancies. We used some strategies to confirm transferability, including recruiting various nurses from different hospitals and institutions and ensured that a variety of people participate in the study.

\subsection{Ethical Consideration}

The institutional review board of Foo-Yin Hospital approved the study (protocol number: FYH-IRB-100-12-04-A). An information sheet explaining the purpose of the research and research activities, description of benefits, and assurance of the data to be taken to protect identities and maintain confidentiality, and acknowledgement that respondent is voluntary was given when recruit potential participants. Consent to participate in the study was implied before proceeding with the data collection, and that refusal or termination of participation will not be jeopardized. This CD and all print materials such as consent forms, demographic data sheet and surveys were stored confidentially and will be kept for a period of three years, and then destroyed.

\section{Results}

\subsection{Characteristics of Participants}

Our participants were 54 nursing staff members who participated in the focus group interviews. There were two men and 52 women, aged 22 to 55 years (average age: 34 years; SD: 10.5). Most of them practiced $(n=51,94.4 \%)$ in the clinical setting as either a registered nurse $(n=44,86.3 \%)$ or a nurse practitioner $(n=7,13.7 \%)$ and had been working from 2 to 21 years (median: 12 years). Participants were working in a wide range of clinical areas, such as medical/surgical wards $(n=27,50.0 \%)$, critical/emergency care $(n=14,25.9 \%)$, pediatrics or obstetrics $(n$ $=5,9.3 \%)$, mental health $(\mathrm{n}=3,5.6 \%)$, aged care $(\mathrm{n}=2,3.7 \%)$ and other settings $(\mathrm{n}=3,5.6 \%)$. Of participants who recorded "Other" for their work settings, this included school, research assistant, and public health bureau. 
Table 1. Participants' Demographic characteristics $(n=54)$

\begin{tabular}{llll}
\hline Demographic characteristics & & Mean & SD \\
\hline Age & & 34 & \pm 10.5 \\
Years of experiences & & 10.17 & \pm 7.07 \\
\hline \multirow{2}{*}{ Gender } & Male & $\mathrm{n}$ & $\%$ \\
& Female & 2 & 3.7 \\
\hline \multirow{2}{*}{ Education } & Bachelor & 52 & 96.3 \\
& Bachelor & 35 & 64.8 \\
\hline \multirow{2}{*}{ Position } & Nurse & 19 & 35.2 \\
& Advanced practice nurse & 44 & 81.5 \\
\hline
\end{tabular}

The purpose of this study was to provide an in-depth description of the following two themes: 1) the reasons for nurses' reluctance to communicate with patients regarding CAM use; 2) the crucial elements for improving CAM communication. Each theme consisted of a number of subthemes that emerged from the data (Table 2). In the next section, verbatim quotes have been included from the raw transcripts as evidence for the findings. The quotes have been translated from the original Chinese. Each participant was switched from name to number instead.

Table 2. Themes and subthemes of the findings

\begin{tabular}{|c|c|c|c|}
\hline Questions & Themes & & iemes \\
\hline \multirow{9}{*}{$\begin{array}{l}\text { Barriers to } \\
\text { communication } \\
\text { about CAM with } \\
\text { patients }\end{array}$} & \multirow{3}{*}{$\begin{array}{l}\text { The scope of nursing practice } \\
\text { regarding CAM is unclear }\end{array}$} & $>$ & Unclear nursing role for CAM practice \\
\hline & & $>$ & Inconsistent acceptance of CAM products in practice \\
\hline & & $>$ & Contradiction to patient medical treatment and safety \\
\hline & \multirow{3}{*}{$\begin{array}{l}\text { Lack of CAM competencies in } \\
\text { communication }\end{array}$} & $>$ & Insufficient knowledge and training of CAM \\
\hline & & $>$ & $\begin{array}{l}\text { Lack of consensus on the best available evidence-based } \\
\text { CAM }\end{array}$ \\
\hline & & $>$ & $\begin{array}{l}\text { Excessive workload leading to disregard for CAM } \\
\text { consultation }\end{array}$ \\
\hline & \multirow{3}{*}{$\begin{array}{l}\text { Unsupportive workplace culture in } \\
\text { the communication of CAM }\end{array}$} & $>$ & Unwritten rules of the workplace \\
\hline & & $>$ & $\begin{array}{l}\text { Absence of communication guideline and } \\
\text { documentation sheet }\end{array}$ \\
\hline & & $>$ & Lack of regulatory system \\
\hline \multirow{8}{*}{$\begin{array}{l}\text { Ways of improving } \\
\text { CAM } \\
\text { communication }\end{array}$} & \multirow{2}{*}{$\begin{array}{l}\text { Awareness of the needs for CAM } \\
\text { education }\end{array}$} & $>$ & $\begin{array}{l}\text { CAM education in Continuing professional training } \\
\text { and school education }\end{array}$ \\
\hline & & $>$ & $\begin{array}{l}\text { CAM education in patients for initiative } \\
\text { communication about CAM }\end{array}$ \\
\hline & \multirow{3}{*}{$\begin{array}{l}\text { Establishment of an organizational } \\
\text { standard for CAM practice }\end{array}$} & $>$ & Standard statements for CAM practice \\
\hline & & $>$ & Guidelines for CAM communication \\
\hline & & $>$ & Unique window for CAM consultation \\
\hline & \multirow{3}{*}{$\begin{array}{l}\text { Engagement of interdisciplinary } \\
\text { teamwork for CAM practice }\end{array}$} & $>$ & Team collaboration for CAM communication \\
\hline & & $>$ & Launching CAM network databases \\
\hline & & $>$ & Resource and referral files for CAM information \\
\hline
\end{tabular}




\subsection{Nurses' Perceptions of Barriers to Communication about CAM With Patients}

Through thematic framework analysis, three major themes were identified in ways in which nurses felt reluctant to communicate with patients regarding CAM use: (1) "the scope of nursing practice regarding CAM is unclear", (2) "lack of CAM competencies in communication", and (3) "unsupportive workplace culture in the communication of CAM".

(1) The scope of nursing practice regarding CAM is unclear

Under this theme, participants highlighted an unclear nursing role for CAM practice, inconsistent acceptance of CAM products in practice, and contradiction to patient's medical treatment and safety as inhibitors for communication about CAM use with patients. Many participants believed that communication about CAM was not entirely the responsibility of the nurse as they said: “...Not everything is our responsibility. Nurses are a part of care team (P15)". Participants suggested that the physician should take more responsibility for this issue:

"Since we are not physicians, they should do the judgement on whether the medicine the patients take is good or bad for the patients." (P37)

They believed that the communication with patients' CAM use is mainly not their responsibility; however, two nurse practitioners indicated that their role required them to assume responsibility for this issue:

"I ignored this when I was a nurse... But I started talking about this issue during the [Nurse Practitioner] training." (P54)

The inconsistent acceptance of CAM products in practice was revealed by many participants. As several participant addressed:

P11: Actually, we accept CAM, but our acceptance is influenced by the way in which it is used.

P8: It is ok if they do not take a CAM product orally. For some products that are fairly invasive, I ask them to discuss with the physicians.

The decision for the acceptance of patients' CAM use among nurses was judging based on whether CAM were internal use or external use. In addition to that, the treatment threatened patient safety and interfered with medical treatment were of concern by many participants.

"If it is not in conflict with medical treatment, then this issue is not of serious concern; so, the patient can continue using it." (P51)

Most participants agreed that "it is necessary to discuss it (CAM)". Participant 25 particularly mentioned the following problem:

"Patients who tend to have UGI (upper gastro-intestinal) bleeding in long-term care unit; so, we always do a nasogastric aspiration to check feed absorption. If family members feed patients Chinese herbs without telling nurses, the color of the extracted liquid will cause errors in the nurses' assessment. It looks like coffee grounds (bleeding) and we make a decision right away - 'NPO'(Nothing by mouth), which will influence patients' nutrition later on."

The concerns did not only focus on the absence of harm for patient, but also center on other patients' safety in ward. Many participants experienced the event like 'burning incantation paper [Taoist magic figures] inside a ward'. As P6 elaborated:

“We usually stop the family when they... there is oxygen around the ward... maybe all the rooms will be gone' (P6)

Based on these descriptions, the scope of nursing practice regarding CAM is unclear as the first barrier to communication about CAM use with patients. This includes unclear responsibility for CAM practice as part of the nursing role and inconsistent acceptance of CAM products in clinical practice.

(2) Lack of CAM competencies in communication

A number of participants raised lack of education/training on CAM, lack of the best available evidence-based CAM and excessive workload as factors having caused negative effects on nurses' motivation to communicate about CAM. CAM is a new concept to many nurses who had never thought about this issue before the interviews.

"What we were taught in the past was unrelated to this; so, CAM is not included in the nursing assessment." (P36)

All nurses cited insufficient knowledge and training in relation to the field of CAM, which made them unwilling to proactively discuss the issue of CAM with their patients.

P18: There are hundreds of CAM... 


\section{P17: We did not learn about CAM during our legitimate medical training.}

P19: We cannot provide them [patients] with appropriate suggestions. This is a difficult situation for us.

To perform a job effectively, what is needed is an integration of knowledge, skills, judgment, and attributes as Participant 51 indicated the importance of competency, which is the capability to perform all necessary tasks without error and display professionalism:

"I believe that you will discuss what you know. If a nurse has obtained this kind of training or course (CAM), then they will be more willing [to communicate about CAM use]."

Many participants have reported being willing to chat with patients regarding the use of CAM; however, they feel that lack of CAM knowledge consequently leads to the fear of communication which causes silence. In addition, lack of empirical findings or support of evidence-based CAM has been revealed in the interviews. P51 pointed out:

"There are only manufacturers [of CAM] who exaggerate the effects, so what we find in practice is not realistic unless there are empirical data."

Almost of all focus groups sited excessive workload as a reason for choosing to avoid this communication because communication about CAM may contribute to heavier workloads and increased overtime:

"The problem is that the nursing workload is really too heavy. [I] just want to go to work and leave the hospital on time. I have tried to discuss [CAM before]. It really took me a long time." (P32)

Therefore, several participants elaborated on the nursing shortage and suggested this issue should be taken up by an advanced practice nurses:

P46: Currently, our nursing manpower is not enough; so, it is necessary that nurse practitioners do this job.

P41: Family members may keep asking questions due to insufficient knowledge; so, the case manager should make time to chat with them.

When nurses experienced burdens due to an excessive workload, they chose to avoid consultation because the needs for communication about CAM is undervalued. In this thematic category, participants illustrated that "insufficient knowledge and training of CAM", "lack of consensus on the best available evidence-based CAM, and "excessive workload leading to disregard for CAM consultation" have become barriers to communication about CAM for nurses.

(3) Unsupportive workplace culture in the communication of CAM

Lack of a communication guideline, a documentation sheet, and a regulatory system, in addition to the unwritten rules of the workplace, have been categorized under "unsupportive workplace culture" causing negative effects on nurses' motivation to communicate about CAM. Many participants mentioned that there are unwritten rules in their units. The discrepancies in attitudes towards patients' CAM use between units within the same hospital have found:

P21: Most of the patients in our unit have terminal liver cancer... We hope they don't take herbal medicine, which may increase the burden [on their liver]...

P22: I started learning about CAM after working in the long-term care department.

P24: We (surgical nurses) won't particularly be concerned with this. So, whether or not to ask about CAM depends on departments.

Attitudes have a powerful influence on behavior. Participants elaborated, saying "nurses' and colleagues' attitudes toward CAM" profoundly influenced their beliefs, feelings, and behavioral tendencies towards communication about CAM. Especially, many participants mentioned that opposition to CAM by physicians is herein contextualized within wider and long-standing professional struggles. "Some physicians disapprove of nurses doing this [communication about CAM]" said P10. Most medical decisions depend on physicians and participants indicated that "... physicians won't allow patients to use [CAM] ...they believe this [prohibition of CAM use] can prevent trouble and be safer." (P49). One participant described how physicians felt being encroached:

"Professionalism is the physicians' territory, if someone flies over [their heads], they feel that their territory has been invaded"(P18).

Other than unwritten rules, there is usually no policy or regulation system to support communication about CAM use with patients. Participant 32 mentioned reasons for the hesitation in communicating about CAM with patients, as follows: 
P32: I probably don't understand the policies of the hospital. There may be a problem if you communicate about CAM. The superintendents of the hospital may disagree with you. We are afraid of being in trouble...

Lack of guidelines and documentation was revealed by many participants as noted: "no suitable tools [for CAM assessment] can be found" (P5, P11, P19, P34, P40, P52). Many nurses thought that this issue should be listed in the logbook or nursing notes, but some people may think it's unnecessary and not important. Everybody has different perspectives. There is no consensus about what and how and where to record patient's CAM use, and many nurses had different perspectives:

P29: If there are side effects..., we will write it in the nursing notes and verbally communicate it to the next shift in-charge.

P30: Things like vitamins won't be noted down. If any name, such as Hedyotis Diffusa, is quite unfamiliar, it will be specially noted it down.

The recording of CAM depended on what they had assessed or if they had observed a potential risk that might harm patients. In fact, many nurses emphasized that the acceptance of CAM use by nurses is considerably higher than that by superintendents and physicians. However, the hierarchical system in a hospital limits nurses' communication about CAM with patients. In this thematic category, unwritten workplace rules, absence of a communication guideline and a documentation sheet, lack of regulation system were barriers to communication about CAM because nurses experienced an unsupportive workplace culture in clinical settings.

\subsection{Ways of Improving CAM Communication}

The ways of improving CAM communication among nurses were discussed in detail with all the groups and three major themes emerged from the analysis of data sources: (1) "awareness of the needs for CAM education", (2) "establishment of an organizational standard for CAM practice", and (3) "engagement of interdisciplinary teamwork for CAM practice".

(1) Awareness of the needs for CAM education

The previous section indicated that nurses were lacking in CAM competencies because of insufficient knowledge and training; therefore, the availability of considerable learning opportunities, information about available resources, and in-service educational programs were proposed in the interviews. Particularly, participants indicated that CAM education should not just happen as part of in-service education, but should start at the school level:

"I think that can be added to the fundamental education, such as internal and surgical medicine. Don't wait until [staff education] ..." (P33)

Further, many participants indicated how teaching this subject could be useful:

"For those who study medical-surgical nursing, evidence-based practice can be added to each [organ] system, in order to enable them to understand how to use it, and then some of the concepts can gradually be developed. In the future, they can further study by themselves and search for evidence. This is probably better for them." (P32)

The teaching strategies suggested by participants, including homework searching for information, conducting systematic reviews, grouping for discussion, reporting on case studies, and practicing simulation-based learning. However, P43 noted that: "No matter how much your school teaches, communication skills require experience." Workshops and seminars are a good way of improving healthcare professionals' consensus on development of CAM practice. In particular, the obligation to hold unit conferences monthly is a great platform for the in-service educational programs to acquire information about CAM communication, as indicated below:

"Special cases can be brought up in the ward meetings...Everybody can come up with creative solutions to the new challenges." (P4)

The previous section indicated that CAM communication is not entirely nurses' responsibility, and that patients are responsible for their own care and treatment. CAM education should be a public movement proposed by participants:

“...healthy people should be educated. Sick people should be educated. Treatment providers should also be educated." (P30)

"We can organize a learning workshop for patients and invite them (pharmacists) to present some restrictions... We can encourage them (patients) to talk on these occasions." (P28)

To promote communication about CAM use among patients, many participants suggested that each department 
should have its own professional education and training. Many participants believed that an on-job-training courses are needed to formally plan and establish the criteria for performance standards, to enable nurses to communicate about CAM use with patients.

(2) Establishment of an organizational standard for CAM practice

Many participants suggested that protocol and guidelines for this communication should be established to enable the provision of a unique window for consultation, such as "a standardized statement" (P50) and "SOPs (Standard Operating Procedures)" (P31). However, this focus group particularly mentioned how it can be promoted:

P15: I think this is up to hospital management, and if hospitals want to address this issue.

P16: Nurses recognize it [the needs for CAM communication] only by policy reinforcement.

The lack of communication regarding guidelines and the lack of a documentation sheet reoccurred throughout the interviews, e.g. "demanding an assessment tool" (P34) and "providing CAM tools" (P1). When discussing when the best time for CAM assessment is, they mentioned several points, such as at "patient hospitalization admission" (P8), during "history taking" (P18), and when "observe abnormalities" (P34).

As P53 concluded this subtheme,

"It [CAM assessment] should be made mandatory. There should be a (assessment) table. We can perform assessments together with this table and a nursing assessment sheet for patient admission."

As in the previous section, participants illustrated that the lack of a communication guideline and documentation, unspoken rules in organization and lack of a regulation system made them feel that the unsupportive organizational culture towards the communication of CAM was a barrier. Governmental and organizational regulations and evaluation along with supervision regulations may affect nurses' engagement in the communication about CAM use with patients.

(3) Engagement of interdisciplinary teamwork for CAM practice

CAM is an umbrella term representing a variety of therapies. Many participants confessed: "There is no way we can understand it (CAM) all" (P35). Team collaboration for CAM communication, launching CAM network databases, and organizing resources for referrals emerged from the interviews regarding improvement of CAM communication. In the enterprise, one of the conditions necessary for successful team collaboration is team members' ability to reach a consensus.

"It (CAM) is the responsibility of entire medical team." (P2)

"Everyone should be in the same boat, paddling in one direction." (P38)

Many participants noted that evidence-based CAM is highly controversial and that there is a substantial lack of consensus among healthcare professionals; therefore, it is difficult for nurses to be confident in communicating about CAM use with patients. Launching a credible CAM network database, which adheres to the principles of evidence-based CAM and critical evaluation, was suggested by many participants: "provide up-to-date, relevant evidence" (P42), “Consists of the best evidence" (P54), "like a guideline of CAM." (P17).

The referral system in the organization is essential for nurses' busy schedule. Participants lacked knowledge regarding CAM, and supportive resources and referral fields for CAM information were mentioned in many focus group discussions:

P16: We can't fully understand it [CAM]; we can only refer patients to special nurses or case managers who work on this exclusively.

P21: ...there should be an assessment [of CAM]. After [completing] assessment, there should be other professionals to contact with the patient.

Prior to making a referral, nurses need to know where to locate valuable reference materials or have information about the availability of resources. As P23 \& P47 said "referral resources in CAM are very few" and "where you can find professionals?" However, P22 took issue with this point and gave an example of how to make a referral:

"I usually refer my patients to a nutritionist (in the hospital). I ask them to bring products and ask the nutritionist to check the composition of ingredients... Therefore, referrals are possible."

The above information highlights the important strategies of improving CAM communication between nurses and patients which have potential useful ways to ensure the safe use of CAM in health care delivery. 


\section{Discussion}

\subsection{Barriers to Communication About CAM With Patients}

Insights gained from this qualitative study found that (1) "the scope of nursing practice regarding CAM is unclear", (2) "lack of CAM competencies in communication", and (3) "unsupportive workplace culture in the communication of CAM" are considered the barriers to communication regarding patient's CAM use in the Taiwanese healthcare context. A previous study found the barriers to CAM communication among Australian nurses, including the context of the nurses' professional work, such as medical dominance and workplace culture, the variety of healthcare providers' attitudes, lack of knowledge about CAM, and lack of time (Hall, Brosnan et al., 2018). These barriers are similar to our findings which have a significant impact on the manner in which nurses communicate with patients about CAM use. Although nurses infrequently initiated discussions on CAM use with patients, they felt very strongly that CAM use should be supervised in some manner.

The inconsistency in attitudes towards CAM within the scope of nursing practice lead nurses to feel ambivalent. Confusions on who should be responsible for CAM communication, what kinds of CAM are acceptable in clinical practice settings and whether CAM is safe use alongside patients' medical treatment were revealed in the interview data. These findings were supported by many studies that culture barriers including personal experience, collegial skepticism, institutional culture; and structure barriers including professional autonomy, knowledge and skills, scope of practice, time, paucity of evidence and contextual relevance were the factors influenced on nurses' decision regarding the practice of CAM (Christina, Abigail, Cuthbertson, \& Whitehead, 2018; Hall, Leach, Brosnan, \& Collins, 2017). A previous study indicated that $80 \%$ of nurses working in acute hospital care $(\mathrm{n}=210)$ sometimes to almost never/never initiated discussion with patients about CAM use. In addition, only $37.5 \%$ of nurses frequently discussed CAM with their nursing colleagues (Hall, Leach, Brosnan, Cant, \& Collins, 2018) which indicated that they may perceive a negative reaction from their colleagues regarding CAM communication. The insufficient workplace support for the implementation of communication about CAM is apparent within hospital culture, where there is a lack of official support and policies to guide communication regarding patients' use of CAM. Indeed, nurse communication regarding patients' use of CAM would be influenced by the system within which nurses work (Hall, Brosnan et al., 2018). However, given the concerns about the safety of certain types of CAM, it is no longer acceptable to disregard CAM in patient care. Lack of discussion regarding CAM use with patients makes it difficult for all healthcare professionals to recognize and report the occurrence of adverse events and drug interactions attributable to CAM, or a combination of CAM and conventional medicines, resulting in missed opportunities to issue essential information to the public.

Despite a growing body of evidence that CAM is flourishing, most nurses tend to ignore CAM literature. Nurses exhibit several gaps regarding knowledge of CAM, attitudes towards CAM practice and communicative behavior about CAM, which may act as barriers to communication about CAM with patients in clinical nursing practice. These findings are also supported by Stub et al. (2016), who reviewed 29 papers and found that lack of scientific evidence, knowledge, and information potentially hinder communication. In this study, the important barriers include the lack of formal rules and standardisation regarding CAM in clinical practice, the absence of pragmatic competence in communication about CAM among nurses, and being unaware of patients' needs, which can hinder nurse-patient communication regarding CAM use and may adversly affect nursing care and patients' disease management. In addition, Chung et al. (2011) have proposed that knowledge and skills in communication with patients related to CAM are becoming increasingly important for all healthcare professionals. Given the fact that nurses are often the first point of contact and care for patients for the longest period within the healthcare system, they should undertake the first patient assessment related to CAM use.

\subsection{Ways of Improving CAM Communication}

Communication about CAM use and conventional treatment between patients and nurses is the key to ensuring the safe implementation of integrated use of all healthcare approaches. To overcome the barriers of CAM communication, interviews suggested the following recommendations: (1) "awareness of the needs for CAM education", (2) "engagement of interdisciplinary teamwork for CAM practice", and (3) "establishment of an organizational standard for CAM practice". In fact, safe and effective management of patients' CAM use heavily depends on collegial and supervisors' support. Across all the interviews, participants indicated the pivotal role of nursing leaders in successfully communicating about CAM in clinical practice. Effective intervention programs for nurses should be supported by the organization, so as to increase their knowledge about CAM. This is supported by Hall et al. (Hall, Leach et al., 2018) who found that level of nursing qualification, completion of any level of CAM training, and workplace environment were profoundly impactful on nurse-initiated discussion of CAM with patients. It is also important to enable nurses to take more initiative in communicating about CAM and 
incorporating the inquiry of CAM use into routine medical histories, medication compliance, and other aspects of care. In addition, the development of standard guidelines for routine assessment of CAM use and an assessment tool for consultation to ensure safe practice is also necessary for all conventional healthcare systems.

As the role of nurses in the practice of CAM has been indicated, it is to address the needs of the whole person, consider each person's values, health beliefs and health experience, and foster a person toward a balanced state of body, mind, emotion and spirit by caring for him/her in a holistic manner and integrating CAM approaches with conventional treatments (American Holistic Nursing Association, 2016). Communication with patients about their CAM use represents an exemplar for advanced nursing practice which nurses should place patients at the center of healthcare decision making, listen what they want with a nonjudgment attitude toward CAM use, engage them in the decision-making process with evidence-based research findings, evaluate the effectiveness of their integrative approaches, and finally, enhance them an optimal state of body-mind-spirit well-being. Findings from this study recommend ways in which nurse researchers may facilitate the application of CAM communication through education, counseling, coaching or other forms of assistance in order to assess the improvement of patients' health outcomes.

\subsection{Limitations}

The unequal male/female ratio of participants is one of the limitations in this study; however; the percentage of male pariticipants in this study was $3.7 \%$, which is similar to the percetage of male nurses $(2.1 \%)$ in Taiwan (Taiwan Department of Health and Welfare, 2018). Another limitation is that the dominant focus group participants might have dominated the discussions. We arranged groups such that members who were well-acquainted with each other, e.g. classmates, but we made certain they worked in different hospitals to prevent domination of the converstaion by those superior and also the spreading of rumours.

\section{Conclusions}

This is the first study to explore nurses' perceptions of barriers to communication about CAM with patients and to determine ways of improving this communication. The findings underline the importance of establishing an evidence-based educational program on CAM to train professionals in the competencies required to provide appropriate CAM communication in different settings, both at the undergraduate level and with experienced healthcare professionals. Moreover, this study also offers many teaching strategies, which may inform future research to assess skill acquisition following the use of a simulation patient and/or role-play training. Furthermore, the establishment of clinical practice guidelines for facilitating and regulating patients' CAM use can improve the quality and safety of health care. Given the importance of patient safety, the first step in the adoption of CAM should be an assessment of how conventional medicines and their CAM counterparts are used by patients, rather than hastening to integrate CAM into clinical nursing practice. Nurses should endeavour to acknowledge patients' CAM use, learn to discuss CAM use with their patients, and do so in an open-minded, respectful manner.

\section{Acknowledgements}

The study was supported by the Ministry of Science and Technology, Taiwan (MOST 101-2314-B-242-001). Our appreciation is extended to the participants who gave so much of their valuable time and thoughts in this study.

\section{Competing Interests Statement}

The authors declare that there are no competing or potential conflicts of interest.

\section{References}

American Holistic Nurses' Association. (2016). Position on the Role of Nurses in the Practice of Complementary \& Integrative Health Approaches (CIHA). Retrieved from http://www.ahna.org/Portals/66/Docs/Committees/Corrected\%20Position\%20Statment\%20on\%20the\%20R ole $\% 20$ of $\% 20$ Nurses $\% 20$ in $\% 20$ the $\% 20$ Practice $\% 20$ of $\% 20$ Complementary $\% 20 \% 20$ Integrative $\% 20$ Health \%20Approaches\%20(CIHA)\%202016.pdf?ver=2017-10-18-101710-343

Chang, H. Y., \& Chang, H. L. (2015). A review of nurses' knowledge, attitudes, and ability to communicate the risks and benefits of complementary and alternative medicine. Journal of Clinical Nursing, 24(11-12), 1466-1478. https://doi.org/10.1111/jocn. 12790

Chang, H. Y., Chang, H. L., \& Siren, B. (2013). Exploring the decision to disclose the use of natural products among outpatients: a mixed-method study. Bmc Complementary and Alternative Medicine, 13. https://doi.org/10.1186/1472-6882-13-319

Chen, K. Y., Wu, M. Y., Yang, P. S., Chiang, J. H., Hsu, C. Y., Chen, C. Y., \& Yen, H. R. (2018). Utilization of 
Chinese herbal medicine and its association with the risk of fracture in patients with Parkinson's disease in Taiwan. Journal of Ethnopharmacology, 226, 168-175. https://doi.org/10.1016/j.jep.2018.08.021

Christina, J., Abigail, W., Cuthbertson, L. A., \& Whitehead, D. (2018). Nurses' Knowledge and Attitudes Toward Complementary and Alternative Medicine for Adult Patients With Cancer in Bandung, West Java, Indonesia: A Qualitative Study. Journal of Holistic Nursing, In press. https://doi.org/10.1177/0898010118811047

Chung, V. C. H., Ma, P. H. X., Tang, T. S. K., Lau, C. H., Kim, J. H., \& Griffiths, S. M. (2011). Do patients tell their clinicians they are using both prescribed and over the counter allopathic and traditional medicines? European Journal of Integrative Medicine, 3(4), E283-E292. https://doi.org/10.1016/j.eujim.2011.09.008

Davis, E. L., Oh, B., Butow, P. N., Mullan, B. A., \& Clarke, S. (2012). Cancer Patient Disclosure and Patient-Doctor Communication of Complementary and Alternative Medicine Use: A Systematic Review. Oncologist. https://doi.org/10.1634/theoncologist.2012-0223

Firkins, R., Eisfeld, H., Keinki, C., Buentzel, J., Hochhaus, A., Schmidt, T., \& Huebner, J. (2018). The use of complementary and alternative medicine by patients in routine care and the risk of interactions. Journal of Cancer Research and Clinical Oncology, 144(3), 551-557. https://doi.org/10.1007/s00432-018-2587-7

Frenkel, M., \& Cohen, L. (2014). Effective communication about the use of complementary and integrative medicine in cancer care. Journal of alternative and complementary medicine (New York, N.Y.), 20(1), 12-18. https://doi.org/10.1089/acm.2012.0533

Foley, H., Steel, A., Cramer, H., Wardle, J., \& Adams, J. (2019). Disclosure of complementary medicine use to medical providers: a systematic review and meta-analysis. Scientific Reports, 9. https://doi.org/10.1038/s41598-018-38279-8

Hall, H., Brosnan, C., Frawley, J., Wardle, J., Collins, M., \& Leach, M. (2018). Nurses' communication regarding patients' use of complementary and alternative medicine. Collegian, 25(3), 285-291. https://doi.org/10.1016/j.colegn.2017.09.001

Hall, H., Leach, M., Brosnan, C., \& Collins, M. (2017). Nurses' attitudes towards complementary therapies: A systematic review and meta-synthesis. International Journal of Nursing Studies, 69, 47-56. https://doi.org/10.1016/j.ijnurstu.2017.01.008

Hall, H., Leach, M. J., Brosnan, C., Cant, R., \& Collins, M. (2018). Registered Nurses' communication about patients' use of complementary therapies: A national survey. Patient Education and Counseling, 101(8), 1403-1409. https://doi.org/10.1016/j.pec.2018.03.010

Hwang, J. H., Kim, Y. R., Ahmed, M., Choi, S., Al-Hammadi, N. Q., Widad, N. M., \& Han, D. (2016). Use of complementary and alternative medicine in pregnancy: a cross-sectional survey on Iraqi women. Bmc Complementary and Alternative Medicine, 16. https://doi.org/10.1186/s12906-016-1167-0

Jou, J., \& Johnson, P. J. (2016). Nondisclosure of Complementary and Alternative Medicine Use to Primary Care Physicians: Findings From the 2012 National Health Interview Survey. Jama Internal Medicine, 176(4), 545-546. https://doi.org/10.1001/jamainternmed.2015.8593

Lincoln, Y. S., \& Guba, E. G. (1985). Naturalistic inquiry. Beverly Hills, Calif.: Sage Publications.

Loquai, C., Schmidtmann, I., Garzarolli, M., Kaatz, M., Kahler, K. C., Kurschat, P., \& Huebner, J. (2017). Interactions from complementary and alternative medicine in patients with melanoma. Melanoma Research, 27(3), 238-242. https://doi.org/10.1097/CMR.0000000000000339

National Center for Complementary and Integrative Health (NCCIH). (2018). Complementary, Alternative, or Integrative Health: What's In a Name? Retrieved from https://nccih.nih.gov/health/integrative-health

Rabiee, F. (2004). Focus-group interview and data analysis. Proceedings of the Nutrition Society, 63(4), 655-660. https://doi.org/10.1079/PNS2004399

Reed, J., \& Payton, V. R. (1997). Focus groups: issues of analysis and interpretation. J Adv Nurs, 26(4), 765-771. https://doi.org/10.1046/j.1365-2648.1997.00395.x

Stub, T., Quandt, S. A., Arcury, T. A., Sandberg, J. C., Kristoffersen, A. E., Musial, F., \& Salamonsen, A. (2016). Perception of risk and communication among conventional and complementary health care providers involving cancer patients' use of complementary therapies: a literature review. BMC Complement Altern Med, 16. https://doi.org/10.1186/s12906-016-1326-3

Taiwan Department of Health and Welfare. (2018). List of Gender Statistics Indicators - Statistics of Nursing 
Graduates and Practicing Nurses in the Department of Health and Welfare. Retrieved from https://dep.mohw.gov.tw/DOS/np-1717-113.html

Wu, H. J., Tai, C. J., Tai, C. J., \& Chien, L. Y. (2019). Symptom severity, symptom interference and use of complementary and alternative medicine among survivors of colorectal and breast cancer after curative treatment in Taiwan. European Journal of Cancer Care, 28(1). https://doi.org/10.1111/ecc.12925

Yeh, M. L., Lin, K. C., Chen, H. H., Wang, Y. J., \& Huang, Y. C. (2015). Use of Traditional Medicine and Complementary and Alternative Medicine in Taiwan. Holistic Nursing Practice, 29(2), 87-95. https://doi.org/10.1097/HNP.0000000000000071

\section{Copyrights}

Copyright for this article is retained by the author(s), with first publication rights granted to the journal.

This is an open-access article distributed under the terms and conditions of the Creative Commons Attribution license (http://creativecommons.org/licenses/by/4.0/). 